\title{
ENTRE L'ÉCRITURE ET L'IMITATION, LA PLACE DU FAIT DIVERS DANS LES PASTICHES DE PROUST $\left.{ }^{*}\right)$
}

\author{
Dr. Inès Saad Mostafa El Sérafi \\ Professeur au département de langue et de littérature Français, \\ Faculté des arts, Université du Caire
}

\section{Résumén:}

A la fois pastiche et fait divers, Pastiches et mélanges restent tributaires d'une vision qui en fait un exercice de style à part entière, (voir Genette, Palimpsestes). Or, une lecture de l'œuvre comme un texte se prêtant à deux écritures, l'une relevant de l'exercice de style (en tant que pastiche) et l'autre appartenant à la fiction populaire (en tant que fait divers) révèle un autre aspect de l'œuvre. Aussi le Flaubert relate-t-il le fait divers en restant fidèle à l'esthétique de la fiction populaire propre à l'écriture d'un 'genre anonyme'. Faisant le contrepied, ou le contrepoids, le pastiche signé SaintSimon représente le cas d'un pastiche né du néant: le lecteur a beau y chercher l'Affaire Lemoine, il ne la trouve pas. Pastiche d'un pastiche, c'est l'exemple d'un texte dans lequel l'imitation n'a d'autre fin qu'elle-même, sorte de pastiche à l'état pur, faisant royalement fi d'un scénario d'origine. Les Pastiches ne seraient pas seulement un recueil de pastiches, Proust s'avérant un écrivain réaliste autant qu'un brillant imitateur. Relevant un double défi, d'imitation et d'invention, en faisant preuve de brio en tant que pasticheur Proust aura du même coup légué à la postérité le seul fait divers qui soit proprement sien.

$\left.{ }^{*}\right)$ Bulletin of the Faculty of Arts Volume 80 Issue 6 July 2020 


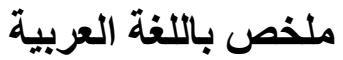

لطالما اعتاد النقاد على قراءة عمل مارسيل بروست

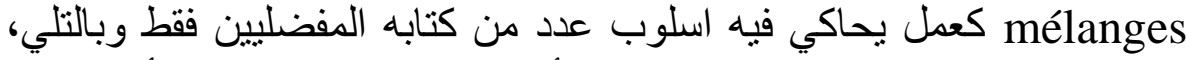

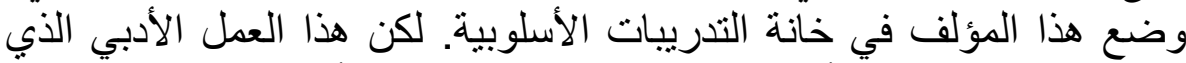

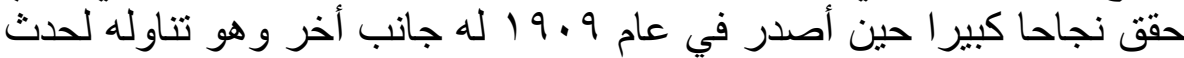

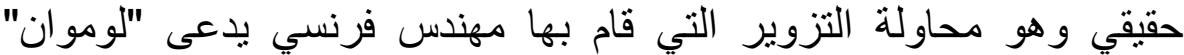

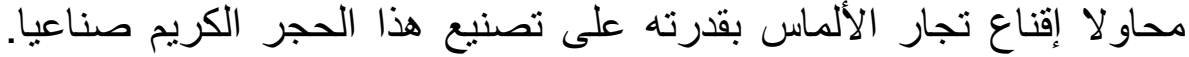

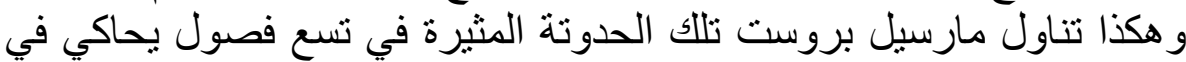
كل فصل الأسلوب الأدبي لكاتب معين. تسعى هذه الدر اسة لإِ لإثبات أن مارسيل

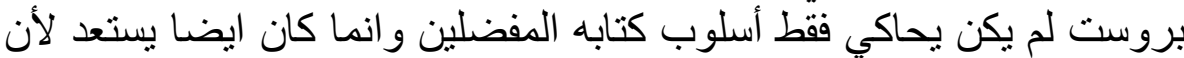

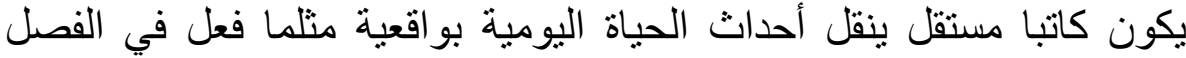

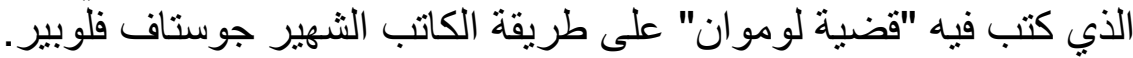

Contenu informationnel appartenant initialement au journalisme, le fait divers entretient avec la littérature des rapports étroits. Ce qui attire les écrivains dans le fait divers est, entre autres, cette promesse de surprise toujours tenue, ce côté insolite d'une information anonyme. Pour un auteur de fiction, romancier ou nouvelliste, à cette dimension s'ajoute une autre: celle de sa délimitation. Dans le fait divers, il s'agit bien d'un fait, d'un acte précis. L'opération de mise en œuvre, laquelle consiste à étoffer les principaux éléments constitutifs de l'événement, transforme celui-ci en narration. À peine intégrant la littérature cette structure minimaliste, scellée par une surprise, devient noyau narratif appelant toutes sortes d'expansion. ${ }^{(1)}$ Inutile de recenser en guise d'exemples tout ce que la littérature doit à une certaine chronique de journaux. Dans ses Pastiches, Proust s'inspirant d'un fait divers, prend un autre parti qui n'est pas sans ambiguïté, convient-il d'ajouter. ${ }^{(2)}$ Depuis janvier 1908, l'escroquerie dont un ingénieur français du nom de Lemoine est l'auteur fait la une des journaux. Une affaire judiciaire intrigante par son côté rocambolesque passionne un public de la presse, habitué au fait divers et y prenant de plus en plus goût. Proust est de ces lecteurs auxquels n'échappe pas un détail de l'Affaire. Cet intérêt de lecteur débouche sur un désir d'écriture, un 
peu particulière: pour la postérité, Proust transforme le fait divers en thème pour des pastiches qui paraissent en forme de recueil de pastiches en 1919. Dès février 1908, ces mêmes lecteurs du Figaro, qui avaient attentivement suivi l'Affaire Lemoine, la relisent diffractée à travers le prisme de l'imitation stylistique. Du 22 février au 21 mars 1908, dans la première page ${ }^{(3)}$ du supplément littéraire du Figaro s'égrènent sept pastiches: Balzac, Faguet, Michelet, Goncourt, Flaubert, Sainte-Beuve, et Renan. L'année suivante est celle d'un dernier pastiche attribué à Régnier. Lors de la parution du recueil, Proust opère encore un ajout en annexant un Lemoine attribué à SaintSimon, 'tiré' de ses Mémoires. En veine d'imitation, l'auteur doublé d'un critique littéraire à l'oreille fine, exercée aux subtilités de style, laisse libre cours à son imagination multipliant les versions qui renvoient à un même fait divers. Ce qui explique que les Pastiches de Proust soient désignés 'Affaires Lemoine' en référence à leur sujet.

À l'origine donc des Pastiches un fait divers qui fait sensation. Dans une première note du recueil, faisant office d'Avant-propos, Proust s'explique sur la nature de l'œuvre sans faire abstraction du sujet à l'origine de sa série de pastiches.

On a peut-être oublié, depuis dix ans, que Lemoine ayant faussement prétendu avoir découvert le secret de la fabrication du diamant et ayant reçu, de ce chef, plus d'un million du président de la De Beers, Sir Julius Verner, fut ensuite, sur la plainte de celui-ci, condamné le 6 juillet 1909 à six ans de prison. Cette insignifiante affaire de police correctionnelle, mais qui passionnait alors l'opinion, fut choisie un soir par moi, tout à fait au hasard, comme thème unique de morceaux, où j'essayerais d'imiter la manière d'un certain nombre d'écrivains. (9)

L'œuvre est donc le résultat d'un double choix: celui d'un thème d'abord, puis d'une écriture. Un fait divers (désigné par litote comme une 'insignifiante affaire de police correctionnelle') et un pastiche ('morceaux, où j'essayerais d'imiter la manière d'un certain nombre d'écrivains', formule où s'inscrit, en creux, l'allusion au recueil de pastiches de Paul Reboux et Charles Müller, À la manière de, publié 
en 1908). Pour l'auteur qui affectionne particulièrement l'écriture pastichielle, en raison de ses vertus cathartiques, critiques ou autres, qui en émaille ses lettres aux amis, ses chroniques de journaux et, plus tard sa Recherche, entre visiblement dans le choix du thème de cette série la passion avec laquelle l'affaire Lemoine fut reçue en son temps par les lecteurs, dont lui. Or, comme il arrive qu'un arbre puisse cacher une forêt, ce qui retient souvent l'attention des critiques dans les neuf textes du recueil c'est leur seul aspect stylistique d'écriture imitative aux dépens d'un autre aspect, à savoir le traitement narratif du fait divers. ${ }^{(4)}$ Les Pastiches de Proust sont appréhendés et lus comme des 'pastiches' plutôt que comme des 'affaires Lemoine'. Le succès du pasticheur serait-il tel qu'il fasse oublier celui de l'écrivain? Proust ne l'a-t-il pas voulu ainsi? N'est-il pas responsable en quelque sorte de cette lecture partielle de son œuvre? Dans sa biographie de Proust, évoquant les pastiches Lemoine, Tadié le dit sans détours: 'On retiendra [...] le mépris de Proust pour la ligne horizontale de l'intrigue: son contenu importe si peu qu'il reste inachevé' et d'ajouter que 'la forme visée par le pastiche' n'est guère plus importante. ${ }^{(5)} \mathrm{Ce}$ que Proust aurait exploité dans son recueil, ce n'est pas autant l'histoire de cette escroquerie qui a tenu en haleine le public du Figaro pendant des mois, que son succès, semble-t-il. Aussi le fait divers cesse-t-il d'être un noyau narratif pour devenir avec Proust un texte, plutôt un prétexte, de réécriture. Dans ses pastiches, la teneur narrative et le côté anecdotique du fait divers semblent relégués au second plan, au même titre que la structure investissant la frappe (et les émotions) qui le caractérise. Les neuf pastiches s'intitulant (plus ou moins) Affaires Lemoine, ne représentent pas neuf versions d'un même fait, mais des aspects de celui-ci au fil des imitations proposées. Ce qui serait la vraie Affaire Lemoine n'est pas neuf fois racontée. Tour à tour assumée par des romanciers, des journalistes, des mémorialistes, des historiens ou des critiques, ses différentes versions portant chacune l'empreinte de l'auteur-modèle du pastiche ne recoupent pas. De sorte que l'on vient à se demander s'il s'agit toujours d'une même 'affaire' au fil des pastiches. Or une autre lecture des Pastiches, tenant compte du fait que leur auteur ait repris neuf fois le même thème, 
permet de nuancer le 'mépris' de Proust pour le fait divers, d'autant plus qu'une trace de celui-ci est repérable dans le recueil. Au gré des transfigurations stylistiques, Proust se serait-il pris d'intérêt pour le fait divers? Celui-ci finit-il par jaillir quelque part dans les Pastiches? Une question s'impose de sitôt: celle de savoir alors lequel des chapitres proustiens respecte (à l'insu de l'auteur?) certains éléments narratifs de l'Affaire quand d'autres la tiennent impunément à distance.

\section{Affaire Lemoine: fait divers ou trame d'écriture ?}

La forgerie de Lemoine inspirant celle de Proust, l'auteur se livre au récit de l'affaire Lemoine en donnant la parole - et la plume à neuf auteurs dont il maîtrise les tics stylistiques ou les idiotismes. Les pastiches proustiens se substituant à l'affaire Lemoine au fil des années, qui cherche à s'informer sur cette escroquerie en apprend d'entrée de jeu sur le recueil polyphonique signé Proust. De quoi s'interroger, dans un premier temps, sur la place qu'occupe le texte d'origine dans l'élaboration d'un pastiche, en l'occurrence celui qui nous intéresse. Selon Genette, le pasticheur ne doit pas toujours disposer de ce qu'il appelle 'un simple scénario, autrement dit (...) un "sujet", inventé ou fourni' pour rédiger un pastiche.(88) Pratiquant un exercice de style en mimant les idiotismes d'un auteur, le pasticheur mettrait même un point d'honneur à créer à partir du néant d'un thème, un texte imitatif (à la manière de...) saturé d'automatismes stylistiques. Genette rappelle que, dans un pastiche, 'l'imitateur a essentiellement affaire à un style, et accessoirement à un texte: sa cible est un style, et les motifs thématiques qu'il comporte (...)'. (89) Ce que l'écrivain imitateur épingle chez son modèle est une certaine vision des choses qui lui inspire leur écriture ${ }^{(6)}$.

La première note des Pastiches ne le laisse pourtant pas penser. D'où l'embarras (ultérieur) de définir l'œuvre. Genette reconnaît dans les Pastiches 'le cas (très exceptionnel) où une série de pastiches [est] composée comme une suite de variations sur un thème unique'. $(131)^{(7)}$ Force est de constater qu'en l'absence d'un 'texte zéro' ou d'un 'degré zéro' (8) du fait divers, il ne peut s'agir de variations sur le même thème, non plus ${ }^{(9)}$. L'originalité des 'Affaires Lemoine' de Proust tient en somme à cette ambiguïté. Le fait divers, éclaté au prisme des écritures, se mue: les pastiches-variations, voulus comme 
tels par leur auteur, se prêtent par un aspect à l'écriture transformationnelle et affichent du coup leur appartenance hybride. Le lecteur des Pastiches est constamment interpellé par cet aspect ambivalent qui se dégage d'un recueil dont les chapitres divers promettent un seul sujet, ce qui l'invite, dans un premier temps, à créer toutes sortes de relations, telles comparaisons, rapprochements ou associations, entre les 'Affaires'. Proust contribue à cet effet en établissant un double contrat de lecture pour son œuvre: en se fixant le style de certains auteurs comme modèle d'une part et en prenant comme point de départ pour ces textes un même scénario, d'autre part. Tout porte à croire que Proust envisage une œuvre ambivalente depuis le début. D'après la note explicative, les Pastiches se lisent comme autant de chapitres à la manière de neuf auteurs que neuf 'manières' d'écrire un seul fait divers inspirées par ces auteurs. Autrement dit, 'l'exercice de thème' qu'est le pastiche se double d'un 'exercice de version' en devenant travestissement ${ }^{(10)}$.

Appréhendant les pastiches Lemoine sous le seul angle de l'écriture pastichielle, Genette les répartit en 'chapitres-hommages' et autres plutôt satiriques, selon un axe qui tient en compte 'la proximité' (affective, faut-il comprendre) par rapport à l'auteur imité. À considérer les Pastiches comme diverses versions d'un (même) fait divers, une autre disposition est possible qui fait cas cette fois de l'intrigue. Aussi proposerons-nous un autre axe de lecture, avec pour critère la proximité également, non pas par rapport à l'auteur pastiché, mais par rapport au sujet choisi, lequel axe intègre désormais le fait divers comme composante des pastiches. De quoi apporter sur ceux-ci un autre éclairage et les pourvoir d'une épaisseur. Est à remarquer le fait que les neuf Affaires en lice ne sont pas toutes de la même veine. Quand trois romanciers mettent en fiction le fait divers, deux mémorialistes en font des 'événements' de leur vie, deux critiques s'en emparent pour leurs chroniques journalistiques et deux historiens l'analysent comme un fait de société qui en dit long sur l'histoire des idées de l'époque. En en faisant un sujet des romans de Balzac et de Flaubert, Proust soumet le fait divers à un sort qui n'a rien d'extraordinaire, notamment au dix-neuvième siècle où il est presque de mode pour les écrivains de s'inspirer des incidents de la vie pour 
écrire des fictions. Les exemples ne manquent pas, de Stendhal à Guy de Maupassant, pour ne citer qu'eux. Le cas d'Henri de Régnier est autre: Lemoine devenu un personnage de roman, de conte plutôt, est décrit par le narrateur, à travers une dramatisation purement symbolique. ${ }^{(11)}$ Le pas est nettement plus grand quand Proust fait de l'Affaire un événement de la vie d'auteurs de mémoires littéraires comme les Goncourt (sous la plume desquels, lui-même, en protagoniste du fait présumé, a failli se suicider) ou d'un mémorialiste doublé d'un historien comme Saint-Simon. Critiques, Émile Faguet et Sainte-Beuve en parlent de seconde main, naturellement, à partir d'œuvres fictionnelles qui auraient adapté le fait divers, ce qui transforme ce dernier en fait littéraire, alors que Michelet et Ernest Renan s'en servent comme prétexte pour des «considérations » sociologiques et morales marquées par l'effusion de l'un et le scepticisme de l'autre.

De toutes les Affaires Lemoine racontées dans les Pastiches, celle faite à la manière de Flaubert se détache de l'ensemble par sa proximité prononcée par rapport au fait divers, et sa place à l'extrémité de l'axe proposé qui prend en compte l'intrigue. Contrairement aux neuf chapitres des Pastiches, les pages attribuées à Flaubert s'inspirent directement de quelques éléments narratifs de l'Affaire Lemoine.

\section{Rester au plus près du fait.}

Pour Genette le pseudo Flaubert représente le cas d'un pastiche à part vu la place particulière que lui accorde son auteur dans ses écrits critiques ultérieurs. Abondamment commenté par Proust (dans sa correspondance ou sa chronique journalistique...), illustration de sa théorie du style comme d'une combinaison bien personnelle des thèmes particuliers de l'auteur et de son expression, le Flaubert est du même coup l'exemple auquel Proust revient plus d'une fois pour expliquer ses propres techniques imitatives (Genette, 112-131), autrement dit sa méthode de travail en tant que critique. Le faux Sainte-Beuve est encore de ces commentaires (au carré) où, à travers le critique, Proust vise l'écrivain. Or dans les Pastiches, l'originalité 
du Flaubert tient également à la relation qu'il entretient avec le fait divers (à la fois en tant que sujet et écriture), et non pas seulement avec le modèle qu'il imite.

En pastichant Flaubert, Proust incruste son Affaire dans une tendance narrative et un courant littéraire qui s'inspirent de la réalité pour produire une fiction. C'est l'observateur social en Flaubert que le pasticheur mime. En épinglant son style et sa vision, son originalité et son impressionnisme, Proust choisit de raconter un épisode bien particulier de l'Affaire: le procès ${ }^{(12)}$. L'allusion au procès traverse les Pastiches plus d'une fois (cas du Michelet, par exemple). Or dans le Flaubert, cet épisode se confond avec le pastiche. Nulle autre Affaire Lemoine ne se prend pour sujet un élément réaliste, quel que soit son degré de vraisemblance ${ }^{(13)}$, du fait divers. Une fiction qui a pour vocation (de par son appartenance générique) de rendre la réalité tient ici sa promesse. Le chapitre s'ouvre sur une description (à laquelle il se résume en somme) du tribunal où a lieu le procès. Il s'agit d'une entrée de plain-pied dans le dernier épisode du fait divers, lorsqu'un verdict tient lieu de mot de la fin, là où tout se joue et se décide. Scène-clef, elle concentre l'essentiel de l'affaire, et sur un plan narratif, elle aurait pu (dû) être une exposition de celle-ci depuis ses débuts, racontée une fois, puis une fois encore, de part et d'autre, entre réquisitoire et plaidoirie. Or ce n'est pas sur la narration que ce Flaubert-là jette son dévolu, mais sur la description. La salle où se tient le procès se métamorphose en un marché public, un bric-à-brac où tout (ou presque) dérive de son emploi d'origine. La scène s'inspire certainement des Comices agricoles de Madame Bovary, et recrée l'ambiance d'Un cour simple qui la pourvoit de quelques éléments proprement exotiques, comme le 'nègre', le 'perroquet' et 'l'orange' désaltérant un public féru d'histoires. Elle est également fondée sur un effet-personnage (caractéristique balzacienne célèbre des descriptions de Flaubert): le personnage du président 'pitre', rentrant à grand peine dans sa 'robe trop étroite', illustre l'étouffement et la pesanteur qui se dégagent de l'endroit. Une foule de personnages secondaires et un amas d'objets hétéroclites (comme Flaubert savait en inventer) créent un 'effet de réel' ancrant le procès, et l'Affaire, dans l'esthétique 'petit 
genre' de la littérature populaire propre au fait divers à laquelle le pseudo-Flaubert reste fidèle. Quand Balzac le métamorphose en l'objet d'une conversation mondaine et aristocratique, évoquée à propos des diamants qu'exhibent marquises et princesses, lors d'un 'rout' dans les salons feutrés d'un hôtel de luxe parisien, Flaubert lui garde toute sa saveur roturière et anonyme. Cet étalage de l'extrême pauvreté du peuple rappelle par son thème une autre forme de la littérature populaire dont l'essor est lié à la presse, comme le fait divers, à savoir le roman feuilleton. Entre les deux formes, Minh Tran Huy perçoit des ressemblances, dont l'origine (naissance dans les chroniques), les thèmes et le cadre (essentiellement populaires).

Rien ne fascine plus [depuis le succès des Mystères de Paris en 1842-1843] que la ville miséreuse, encrassée, dévoratrice, peuplée de figures de l'ombre, fruit d'une urbanisation accélérée (...) la ville et ses ténèbres, ses 'mystères', sont devenus le support d'une immense peur sociale et un extraordinaire lieu de fantasmes - (48)

Comme le roman feuilleton dont il est une source, le fait divers entretient un rapport avec les basfonds et les 'figures de l'ombre'. Petite histoire 'des gens qui n'ont pas d'histoire' (Goncourt à propos du fait divers, préface de La Faustin), anecdote, 'inclassable' et 'rebut inorganisé' appelé à se voir confiné dans les Varia, le fait divers est un 'art de masse' (Barthes). ${ }^{(14)}$ Les foules s'y reconnaissent, sont ses spectateurs et ses acteurs. Flaubert se garde de dépayser l'intrigue. Le choix de ses mots en témoigne: de ces 'intimités qui s'ébauchèrent' entre des inconnus empressés de voir l'accusé, à l'effet 'de décoratif et de vulgaire' qui se dégage de la personne du président, en passant par cet homme du peuple qui 'par amour pour la popularité' partage 'sur un journal' le petit bien qu'il a 'en s'excusant'. (19-22). Sa description de la salle 'officielle' où trônent les portraits de deux présidents, est sans appel. Carnavalesque, elle se transforme en une mise en scène où, chemin faisant, la sphère officielle est tournée en dérision.

Pour finir, il attesta les portraits des présidents Grévy et Carnot, placés au-dessus du tribunal; et chacun, ayant levé la tête, constata que la moisissure les avait gagnés dans cette salle officielle et 
malpropre qui exhibait nos gloires et sentait le renfermé. Une large baie la divisait par le milieu, des bancs s'y alignaient jusqu'au pied du tribunal ; elle avait de la poussière sur le parquet, des araignées aux angles du plafond, un rat dans chaque trou...(20)

Le tribunal, salle fourre-tout où tout ce qui est hétéroclite a sa place, est le lieu idéal de toutes les mésalliances et de toutes les allusions. Se prêtant à la charge, le discours juridique est disqualifié. Il est tantôt emphatique, monotone ou insignifiant, tantôt pathétique, médiocre ou prétentieux, toujours comique.

...l'avocat de Werner prononçait sa plaidoirie. Il avait débuté sur un ton d'emphase, parla deux heures, semblait dyspeptique, et chaque fois qu'il disait 'Monsieur le Président' s'effondrait dans une révérence si profonde qu'on aurait dit une jeune fille devant un roi, un diacre quittant l'autel. Il fut terrible pour Lemoine (...). L'avocat de Lemoine répliquant, fut bref. Mais il avait un accent méridional, faisait appel aux passions généreuses, ôtait à tout moment son lorgnon. (20)

Les figures de l'emphase qui permettent à l'ironie 'flaubertienne' de viser l'autorité constituent une autre composante du fait divers. Désignant 'à la fois un événement et une manière d'en parler, une façon', un effet de mise en scène ressortit nécessairement à l'écriture du fait divers, d'où le goût des 'superlatifs, phrases nominales et points d'exclamation'. ${ }^{(15)}$ Après le président et les deux avocats qui s'étaient exprimés d'une façon on ne peut plus théâtrale, vient le tour de l'accusé.

...un murmure s'éleva, deux parapluies tombèrent: on allait entendre à nouveau l'accusé. Tout de suite les gestes de colère des assistants le désignèrent; pourquoi n'avait-il pas dit vrai, fabriqué du diamant, divulgué son invention? (21)

Or l'accusé ne parlera pas. À sa place d'autres voix se feront entendre, à travers le silence. L'audience laisse tomber son verdict: Lemoine est coupable. S'il avait 'dit vrai', il aurait fait la fortune de tous ces miséreux et pas seulement la sienne. Juge-t-on Lemoine pour la même raison de part et d'autre de la salle? Reste-il toujours un 
escroc, ce philanthrope de Lemoine qui aurait donné en partage son secret de diamants industrialisés et aurait réalisé -on ne sait trop comment- des rêves de 'confort' et d'influence'? L'Affaire Lemoine s'avère une affaire d'aspirations petites bourgeoises toutes frustrées, de bovarysme en somme. Loin du peuple devenu juge le temps d'un procès, la digression sur les attentes déçues met en scène le côté mélodramatique $\mathrm{du}$ fait divers appelé à une consommation 'communautaire' (Hamon) ${ }^{(16)}$. Cette place centrale que Flaubert confère à Lemoine, les rêves attachés à son délit et la frustration collective qu'il suscite par son échec, transforme le personnage en héros, une sorte de 'justicier criminel' (Minh Tran Huy) ou de redresseur de torts, comme en regorgent les fictions populaires dont le roman feuilleton. Selon Barthes, le fait divers est une information fondée sur une déviation, information qui, par sa structure causale 'trouble', prise ce genre d'effets où le hasard ne cesse de jouer et les forces extraordinaires d'intervenir.

...le fait divers est riche de déviations causales (...) la cause révélée est d'une certaine manière plus pauvre que la cause attendue (...) il y a en effet dans ce genre de relation causale, le spectacle d'une déception: paradoxalement, la causalité est d'autant plus notable qu'elle est déçue. (446)

Proust recrée cet effet d'incongruité propre au fait divers, où tout tient toujours à rien ('petites causes, grands effets', Barthes) par ce genre de déplacement dont seul est capable le réalisme ironique d'un Flaubert: les victimes de la forgerie ne sont plus les riches de la De Beers, mais des gens ordinaires crument déçus. Mais leurs projets échafaudés ne sont que délire auxquels ils s'abandonnent, et leurs rêves fantaisistes sont teintés du moralisme inséparable d'une sensibilité pathétique, caractéristique du fait divers.

À ceux-là, l'excès de leur détresse ôtait la force de maudire l'accusé ; mais tous le détestaient, jugeant qu'il les avait frustrés de la débauche, des honneurs, de la célébrité, du génie ; parfois de chimères plus indéfinissables, de ce que chacun recélait de profond et de doux, depuis son enfance, dans la niaiserie particulière de son rêve. (22) 
La 'niaiserie' et les 'chimères indéfinissables' donnent au pseudoFlaubert sa dernière note. D'une affaire de diamants, Proust brode un fait divers, autrement dit une affaire du peuple. Les cacophonies de Flaubert (Genette), celles qui font son style et son originalité, égrènent généreusement son 'Affaire Lemoine': les phrases 'maçonnées' (Proust) ${ }^{(17)}$ et la lourdeur qu'elles dégagent, la singularité de l'écriture par laquelle s'exprime la singularité de la vision (Genette, 126), l'emploi rebelle des conjonctions, dont le fameux 'et', jamais à sa place, qui ne coordonne pas mais disloque (Genette), et des modes verbaux (notamment du passé), sans parler de l'effet de 'rupture' qui constitue, selon Genette, le vrai 'flaubertisme'. Une thématique bien flaubertienne est épinglée par Proust lorsqu'il retient de son modèle le subjectivisme et 'le primat de l'impression' (Genette), cette 'tristesse' née de 'la rupture des habitudes et de l'irréalité du décor' (Proust) ${ }^{(18)}$, son concept du temps et la fonctionnalité des personnages secondaires. Tout ce qui fait de l'écriture de Flaubert une 'prose à la Courbet'(19) trouve place dans le pastiche. L'ensemble est savamment façonné par l'esthétique d'une forme de la littérature populaire qui s'exprime par les émotions fortes et les petites causes, les projets gigantesques et les rêves fantaisistes, les grands tenants et les minces aboutissants. De toutes les formes littéraires qui s'offrent dans les Pastiches, le fait divers est livré aux seuls soins du 'Flaubert' qui, même à travers le choix d'une description-charge, lui préserve toute sa saveur et le pourvoit d'éléments relevant autant de la thématique de la forme que de celle de l'auteur. Aussi le choix du tribunal comme 'scène' ne peut être gratuit: un des épisodes (et des lieux) familiers du fait divers, l'espace participe à la création d'une illusion réaliste.

Si les autres pastiches du recueil retiennent, plus ou moins, des détails ou des aspects du fait divers, ils ne manquent pas de les déployer aux fins de l'écriture imitative. Ne hantant pas le recueil de la même façon, l'Affaire Lemoine n'entre pas par la même porte ni ne se situe à la même distance par rapport à chacun des auteurs imités. Se saisissant directement du fait divers, Balzac et Flaubert seraient, par exemple, en relation plus directe avec celui-ci qu'un Sainte-Beuve ou un Faguet qui, en critiques, écrivent sur des auteurs ayant traité 
narrativement le fait (le cas du couple Sainte-Beuve-Flaubert est unique, Proust nous mettant sous les yeux à la fois le pseudo-roman et le supposé métadiscours qu'il inspire) et sont donc en rapport indirect avec ce dernier. L'allusion au diamant auquel le narrateur de Régnier 'ne trouve pas de beauté' (31) et, in fine, la symbolique du nez de Lemoine qui coule, ne suffit pas pour faire de ce dernier le héros d'une filouterie (33-34). Telle que l'exposent Michelet et Renan, l'escroquerie, 'épisode de la grande lutte de la richesse contre la science'(40), est prise dans des digressions, quand elle n'en est pas une elle-même.

...le juge Le Poittevin, avec un tact exquis, ne le laissait pas poursuivre, lui indiquait d'un mot, parfois d'une plaisanterie un peu vive, toujours contenue par un rare sentiment de la mesure, qu'il ne s'agissait pas de cela, que la cause était ailleurs. (47)

L'Affaire est racontée par bribes ou par prétérition ('Je raconterai quelque jour cette magnifique affaire Lemoine dont aucun contemporain n'a soupçonné la grandeur') et ne tarde pas à se transformer en signe du 'matérialisme grossier avec lequel devra compter de plus en plus celui qui prétend se mêler des affaires de l'humanité' (47). Le Journal des Goncourt, dispositif hybride relevant à la fois de la chronique littéraire et de l'écriture personnelle, nous présente le fait divers comme un projet littéraire qui n'eût pas lieu, et les deux frères comme des auteurs à l'œuvre (tel Proust s'essayant plus d'une fois dans les Pastiches). Entre potins, ragots et médisances, rêves désagréables et pressentiments de mauvais augure qui tous se réalisent, les auteurs, devenus critiques entre temps, livrent leur secret de la fabrication romanesque. De quoi faire de ce pastiche encore une évocation en seconde main du fait divers. Un dernier pastiche, également dernier en date, manque à cette liste: le pseudo SaintSimon. Pastiche complexe en soi, Saint-Simon retient l'attention par l'effet de distance qu'il marque par rapport au fait divers. Contrairement au 'Flaubert' respectueux aux éléments de l'intrigue et à l'esthétique d'une écriture, le 'Saint-Simon' arbore une indifférence à l'Affaire Lemoine qu'il réduit du coup à une simple trame d'écriture. De quoi faire de cette l'Affaire-là la plus éloignée du fait divers pour 
des raisons aussi bien stylistiques que thématiques. Aux antipodes du romancier se situe donc le mémorialiste dont le pastiche dépayse on ne peut plus le fait divers en l'insérant dans une forme littéraire pourtant non-fictionnelle.

\section{Quand Saint-Simon snobe Lemoine.}

Le chapitre le plus long du recueil, dont on nous promet 'une suite', est à la fois le plus ancien et le dernier en date à s'annexer aux Affaires Lemoine. À première vue, le pseudo-Saint-Simon fait figure d'un intrus sur la liste proustienne des Affaires Lemoine. Proust insère le fait divers dans les pages de Mémoires qui regorgent de noms d'une autre époque, de Mémoires 'royales' où tout, même les menus faits, gravitent autour d'un Roi-Soleil. Ce serait pour cela pourtant que l'Affaire Lemoine chez Saint-Simon interpelle: le lecteur n'a de cesse de la chercher dans un texte qui la promet et oublie commodément de s'en acquitter. L'Affaire Lemoine 'Dans les Mémoires de Saint-Simon' se présente d'emblée comme un cas de pastiche unique et ce pour plusieurs raisons. Encore un pastiche admiratif (Genette) dont la petite histoire se confond, par certains moments, avec la genèse de la Recherche et son développement. Singulièrement antérieur au fait divers, le pseudo-Saint-Simon remonte au 18 janvier 1904, quand Proust -grand lecteur de Saint-Simon qu'il admire particulièrement publie au Figaro une 'Fête chez Montesquiou à Neuilly (Extrait des Mémoires du duc de Saint-Simon)' sous le pseudonyme d'Horatio. Le pastiche est une chronique mondaine, comme en foisonnent aussi bien les Mémoires du Grand Siècle que les journaux de l'époque de Proust. Depuis 1909 l'auteur travaillait au regroupement des huit pastiches Lemoine en une œuvre et, parallèlement, à l'adaptation d'un neuvième, attribué à Saint-Simon, à partir de la chronique de 1904. Ce n'est qu'en 1919, lorsque les Jeunes filles en fleurs de Proust sont publiées, qu'un recueil de neuf affaires Lemoine est entre les mains des lecteurs. Le succès du roman profite visiblement aux Pastiches. Pourvu d'une histoire antérieure à celle des pastiches et du fait divers, le pseudo Saint-Simon est à juste titre le pastiche d'un pastiche. La dernière Affaire Lemoine se distingue de l'ensemble du recueil par deux types d'inventions, ou deux torsions narratives, qui achèvent 
d'éconduire le fait divers. La première invention consiste à remodeler un texte tout fait, la chronique antérieure, en lui annexant un élément relatif au fait divers. Or cette annexion fait pâle figure et s'insère comme autant un menu détail à propos d'un personnage on ne peut plus saint-simonien, à savoir le duc d'Orléans.

Le Moine, qui avait passé sa vie, enterré dans la crapule la plus obscure et ne connaissait pas à la cour un homme qui se put nommer, ne sut pourtant à qui s'adresser pour rentrer au Palais Royal ; mais à la fin, la Mouchi en fit la planche. Il vit M. le duc d'Orléans, lui dit qu'il savait faire du diamant, et ce prince, naturellement crédule, s'en coiffa.(59-60)

Ouvert à l'actualité mondaine de Proust, grouillant de personnages qui serviront de modèles à la Recherche, le pastiche ne tient à l'affaire des diamants que par un fil, celui de la crédulité naturelle du duc d'Orléans, un des personnages principaux des Mémoires de Saint-Simon, et l'allusion à une 'crapule' du nom de 'Le Moine'. Entre histoires de rangs et de préséances, de 'familles' et de 'naissances', de prétentions incongrues, et d'étiquette bafouée, de généalogie et toutes sortes 'd'alambics' d'usages (Proust), Lemoine prend le temps de devenir Le Moine, pendant que le cadre de l'Affaire est transposé de la 'cour de justice' à la 'cour royale'.

Une deuxième invention ressortit à l'écriture du pastiche en tant qu'exercice d'imitation stylistique. L'essentiel de Saint-Simon étant à prendre, un effet d'anachronisme se dégage forcément du dernier chapitre des Pastiches. Proust commence par coiffer l'affaire incorporée aux Mémoires d'un appareil paratextuel et intertitulaire articulé en forme de sommaire où l'on voit que Lemoine s'écrit Le Moine. Ceci achève de conférer à l'Affaire 'Dans les Mémoires de Saint-Simon' un aspect transpositionnel qui opère par les archaïsmes, aussi bien thématiques que stylistiques, inspirés par le choix de l'auteur imité. Au nombre de ces archaïsmes thématiques qui le mènent à réinventer presque le fait divers, le développement par lequel Saint-Simon retrace les débuts de l'affaire.

Les affaires du Mississipi avaient tourné court et le duc 
d'Orléans venait, contre mon avis, de rendre son inutile édit contre les pierreries. Ceux qui en possédaient, après avoir montré de l'empressement et éprouvé de la peine à les offrir, préfèrent les garder en les dissimulant, ce qui est bien plus facile que pour l'argent, de sorte que malgré tous les tours de gobelets et diverses menaces d'enfermerie, la situation des finances n'avait été que fort peu et fort passagèrement améliorée. Le Moine le sut et pensa faire croire à $\mathrm{M}$. le duc d'Orléans qu'elle le serait s'il le persuadait qu'il était possible de fabriquer du diamant. Il espérait du même coup flatter par là les détestables goûts de chimie de ce prince et qu'il lui ferait ainsi sa cour. (59)

Un fait divers ne pouvant constituer le sujet d'une page de SaintSimon, Proust se devait d'inventer une genèse de l'affaire qui serait compatible avec la thématique des Mémoires. Lemoine devait migrer, non seulement au gré des époques, mais à travers les formes et dans les sphères de la narration. 'Le Moine' à qui Proust ôte même le primat de l'initiative est chassé hors de l'intrigue. Lemoine n'est plus l'auteur de son Affaire. Un édit orléaniste 'inutile' et 'contre l'avis' de SaintSimon aurait vaguement inspiré la forgerie à Lemoine, sans plus. D'ailleurs, tout semble confirmer le fait que le 'délit' proprement dit n'eut pas lieu. L'affaire est un sujet de conversation: elle devient ce qu'on en dit. Ni Lemoine ni son escroquerie ne constituent le sujet de cette chronique-là, mais des histoires de généalogies et de prétentions à des privilèges hors de portée.

Je tâchai d'arraisonner M. le duc d'Orléans, je lui représentai l'injure qu'il faisait non seulement aux ducs, qui se sentiraient tous atteints en la personne du duc de Gramont, mais au bon sens, en laissant le prince Murat (...) avoir pendant le parvulo de Saint-Cloud, la main qu'il se garderait bien de ne pas exiger ensuite à Versailles, à Marly, et qu'elle servirait de véhicule à l'Altesse, car on sait où conduisent ces sourdes et profondes menées de princerie quand elles ne sont pas étouffées dans l'œuf. (...) L'affaire du Moine ne touchait pas à des intérêts si vitaux pour la France. (67-68)

L'affaire du Moine', à résonnance déjà autre, est ravalée au rang 
d'incongruité, qu'il faut vite chasser. Inféodée à la description du duc d'Orléans, elle est une preuve d'aveuglement' et d'ensorcellement', et atteste de cette gaucherie dont Saint-Simon fait le trait principal de son portrait.

À relever, un autre archaïsme thématique, à savoir le système de personnages où se mêlent personnages de la société de Proust, appelés plus tard à passer dans la Recherche, à des noms de la cour de Louis XIV, système où les personnages de fiction côtoient ceux de l'histoire. En contrefaisant la prose de Saint-Simon, Proust n'ancre pas l'affaire une fois pour toutes dans un cadre temporel ancien en la faisant remonter à quelques siècles plus tôt. C'est dire qu'au lieu d'une reconstitution historique du fait, il opte de façon ludique pour un jeu de télescopage et de confusion chronologique entre le présent du pasticheur et le passé du modèle pastiché. Subtilités temporelles qui fonctionnent comme autant de faux-fuyants narratifs en permettant force projections et allusions à son actualité.

Mlle Asquith, qui était probablement la plus intelligente d'aucun, et semblait une de ces belles figures peintes à fresque qu'on voit en Italie, épousa le prince Antoine Bibesco, qui avait été l'idole de ceux où il avait résidé. Il était fort l'ami de Morand, envoyé du Roi auprès de leurs Majestés Catholiques, duquel il sera souvent question au cours de ces Mémoires, et le mien. Ce mariage fit grand bruit, et partout d'applaudissement. Seul, un peu d'Anglais mal instruits, crurent que Mlle Asquith ne contractait pas une assez grande alliance. Elle pouvait certes prétendre à toutes, mais ils ignoraient que ces Bibesco en ont avec les Noailles, les Montesquiou, les Chimay, et les Bauffremont qui sont de la race capétienne...(68-69)

Les amis de Proust peuplent Saint-Cloud. L'occasion, semble-til, de reconnaître des dettes ou des filiations littéraires et de témoigner d'anciennes amitiés. Noyé dans l'épisode mondain où en surimpression se détachent les figures de la société parisienne contemporaine de Proust et de sa Recherche sur un canevas du Grand Siècle, le goût du duc d'Orléans pour la chimie (à propos duquel Le Moine est évoqué dans le pastiche) est à peine un alibi. Le récit se 
dissout dans la description du parvulo de Saint-Cloud qui, à son tour, est un calque des soirées mondaines auxquelles assiste Proust. Dans son étude du pastiche de Saint-Simon par Proust, Jean Milly ${ }^{(20)}$ souligne aussi bien que les idiotismes de l'auteur imité, ce qui serait un 'proustisme' du texte: portraits - inspirés de l'art de Saint-Simon de personnages-modèles de sa fiction, hommages rendus à des amis, mondanités reprises dans la Recherche, etc. De quoi faire du pseudoSaint-Simon le premier autopastiche de Proust ${ }^{(21)}$ en somme.

La chronique élogieuse que fut la 'Fête chez Montesquiou à Neuilly (Extrait des Mémoires du duc de Saint-Simon)' hante le pseudo Saint-Simon du 'Moine' qui devient du coup un pastichepalimpseste. Ni Verner, ni De Beers, ni faux, ni procès, ces Mémoires de Proust réduisent l'Affaire à un simple prétexte non pas d'écriture cette fois, le pastiche de Saint-Simon datant de quelques années, mais de publication en œuvre. Revenu à la charge avec Lemoine, Proust garde à la chronique mondaine son assaisonnement stylistique qu'il fait revivre, tout en retenant de son modèle 'la liberté chaotique de la langue, dit Genette, et le prodigieux enchevêtrement syntaxique' (111). Proust emprunte à Saint-Simon ses images ainsi que l'emploi particulier de certains termes (tel 'bonhomme'(22)), sans parler des mots propres à une époque qui remonte à trois siècles. Le pastiche des Mémoires se clôt par une phrase-pirouette qui livre son secret et dénonce ce qu'elle prétend cacher.

Mais cette digression sur les titres singuliers nous a entraînés trop loin de l'affaire du Moine. (82)

Or cette digression, c'est tout le pastiche. C'est l'affaire Lemoine telle que la raconte Saint-Simon, affaire d'où le fait divers snobé est subtilement éconduit au prix d'une fidélité à un autre texte, le pastiche antérieur, à la définition du genre mémorialiste où le drame populaire, vraie peinture de genre par rapport à l'Histoire, n'a pas droit de cité, et à l'esthétique d'un auteur imité. Du fait divers, le pseudo Saint-Simon garde seulement ces mots que Proust conjugue à la thématique de son modèle: diamant, et forcément Le Moine dans une typographie Grand-Siècle. Un fait qui n'a plus rien de 'divers' 
raconte une autre histoire. Cette 'passion' que l'affaire correctionnelle avait suscitée chez les lecteurs de journaux, celle-là même qui était à l'origine des pastiches, n'a pas trace dans le Saint-Simon, pastiche autarcique par excellence.

À travers des exercices ludiques de style, voulus tantôt comme une critique vivante, tantôt comme la purgation du charme d'un auteur, un fait divers est parvenu, transfiguré, jusqu'aux lecteurs d'un autre temps. Relevée par la veine fantaisiste d'un écrivain-pasticheur, l'Affaire Lemoine est désormais présente, mais sous un autre jour. Que cela soit en restant au plus près de l'intrigue ou en la snobant, les Pastiches, qui confèrent pérennité à un genre anonyme et de mauvaise presse par le biais de la littérarité, font sort au fait divers en maintenant une ambiguïté que les critiques ont tôt fait d'éluder. Les neuf Affaires de Proust ne sont pas toutes pareillement Lemoine. Une lecture les réduisant au seul statut d'écriture 'au second degré' les avait mises à pied d'égalité. Or, à ce prix un autre aspect du recueil passe souvent inaperçu. L'Affaire Lemoine survit à son époque grâce à une œuvre qui confirme la réputation de Proust en pasticheur chevronné mais aussi en écrivain réaliste, tel qu'il se manifeste dans le pseudoFlaubert. Un pastiche se prenant pour double modèle le style de l'auteur pastiché et l'esthétique du fait divers se transforme en exercice d'écriture hybride, d'imitation et d'invention. En mimant les idiotismes stylistiques de Flaubert, Proust semble avoir les yeux rivés sur une forme d'écriture fortement stylisée (contrairement à ce qu'on croirait à première vue, souligne Minh Tran Huy) dont le propre est de produire de fortes impressions. Le Flaubert est né de ce double souci. Proust restitue à l'Affaire Lemoine son univers clos d'être immédiat, total, qui ne renvoie (...) à rien d'implicite' (Barthes) et la pourvoit d'une mise en scène propre au fait divers, s'aidant d'effets de style proprement flaubertiens. Les pages attribuées à Flaubert sont saturées des automatismes de l'auteur et de ceux d'une forme anecdotique et anonyme. Sous le masque du pasticheur un écrivain réaliste ne pointet-il pas son nez? Le contrat de pastiche (d'ailleurs respecté) ne devientil pas pour Proust un prétexte pour se prêter à l'écriture d'une forme de la littérature populaire? S'essayant à l'écriture romanesque, Proust, 
le mondain, se laisse inspirer par une affaire judiciaire, le temps d'un pastiche. De quoi faire de 1'“'Affaire Lemoine" par Gustave Flaubert' le seul fait divers écrit à la manière de Proust. 


\section{Notes:}

(1) Voir Daniel Grojnovski, Lire la nouvelle (Paris: Dunod, 1993), pp.44-57.

(2) Marcel Proust, Pastiches et mélanges, (Paris: Gallimard, 1970).

(3) Se faire publier dans la première page du supplément littéraire du Figaro revêt une importance particulière aux yeux de l'auteur. Proust est soucieux de la façon dont se présentent ses pastiches auxquels il voudrait attirer toute l'attention du lecteur. Le pastiche, 'genre assez secondaire', avoue-t-il dans une lettre datée du 11 mars 1908, doit être relevé par une place en vue. Or c'est le fait divers, micro-histoire des foules, qui semble prendre ainsi sa revanche en désertant sa place habituelle dans les quotidiens, quelque part dans des rubriques à l'écart, souvent anonymes.

(4) Dans Palimpsestes Gérard Genette l'analyse comme l'exemple d'une œuvre écrite sur le mode du pastiche, Luc Fraisse en parle à deux reprises dans le seul cadre de cet aspect du génie proustien qu'est l'imitation, quant à Minh Tran Huy parlant de Proust et de fait divers, elle évoque Sentiments filiaux d'un parricide et pas Lemoine. Voir à ce propos Gérard Genette, Palimpsestes, (Paris: Seuil, 1982), Luc Fraisse, 'Proust: philosophie du pastiche et pastiche de la philosophie', Revue d'histoire littéraire de la France, 1, (2012), pp. 63-75, et 'Les pastiches de Proust éclairés par l'histoire du genre', Romanische Forschungen, 124-1, (2012), pp. 51-60, et Minh Tran Huy, Les écrivains et le fait divers : une autre histoire de la littérature,(Paris: Flammarion, 2017).

(5) Jean-Yves Tadié, Marcel Proust, (Paris: Gallimard, 1996), p.605.

(6) À ce propos, Genette rappelle que Proust concevait le style comme autant une vision qu'une manière d'écrire, des thèmes aussi bien qu'une technique.

(7) Le (faux) parallèle avec les Exercices de style de Raymond Queneau s'impose évidemment.

(8) Nous empruntons ici une expression de Gérard Genette, employée par lui à propos des Exercices de style de Raymond Queneau. Voir Genette, Palimpsestes, p.133.

(9) D'ailleurs que serait le texte initial d'un fait divers: ce récit que les chroniques des journaux racontent avec plus ou moins les mêmes détails ou celui de l'acte lui-même?

(10) Voir la terminologie, et la typologie, de Genette, Palimpsestes, p.88.

(11) Le décor et les personnages du pastiche sont ceux du conte Le Trèfle noir, publié en 1895.

(12) Le choix du procès n'est pas gratuit et reste profondément en relation avec le fait divers, vu que c'est bien le déroulement du procès de Lemoine que les lecteurs du Figaro suivent avec cette 'passion' qu'il avait mentionnée au début de son recueil.

(13) Nous savons ce que Proust pense de cette 'vraisemblance' dont il fait le critère d'une argumentation étriquée et mesquine (Genette) élaborée par le pseudo Sainte-Beuve contre les pages pessimistes de Flaubert.

(14) Roland Barthes, 'Structure du fait divers', Essais Critiques in Euvres Complètes, éd. Éric Marty, nouv. éd., 5 vols, (Paris: Seuil, 2002), II, pp.442-51.

(15) Minh Tran Huy, p.18 
(16) Philippe Hamon, Introduction, 'Fait divers et littérature', Romantisme, 27,97, (1997), pp.7-16.

(17) Marcel Proust, 'À propos du "style" de Flaubert' in Chroniques, (Paris: Gallimard, 1927), pp.193-211.

(18) Ibid., p.199.

(19) L'expression est de Proust. 'Proust et le style de Flaubert', lettre à Léon Daudet, Les Amis de Flaubert, 31, (1967), p.31.

(20) Jean Milly, 'Un pastiche de Proust: "L'Affaire Lemoine dans les Mémoires de Saint-Simon"', Cahiers Saint-Simon No5, 1977, pp.17-22.

(21) Sur l'autopastiche proustien, voir entre autres, Genette, Palimpsestes, pp.135138 et Emily Eells 'Proust à sa manière', Littérature, No46, 1982, pp.105-123.

(22) Pastiches et mélanges, p.9. 


\section{Liste bibliographique}

\section{Corpus}

Marcel Proust, Pastiches et mélanges, (Paris: Gallimard, 1970).

\section{Ouvrages critiques}

Roland Barthes, 'Structure du fait divers', Essais Critiques in Euvres Complètes, éd. Éric Marty, II, Seuil, 2002, pp442-451.

Emily Eells, 'Proust à sa manière', Littérature, No46, 1982, pp.105123.

Luc Fraisse, 'Proust: philosophie du pastiche et pastiche de la philosophie', Revue d'histoire littéraire de la France, 1, 2012, pp. 63-75.

'Les pastiches de Proust éclairés par l'histoire du genre', Romanische Forschungen, 124-1, 2012, pp. 51-60.

Gérard Genette, Palimpsestes, Seuil, 1982.

Daniel Grojnovski, Lire la nouvelle, Dunod, 1993.

Philippe Hamon, Introduction, 'Fait divers et littérature', Romantisme, 27,1997, pp.7-16.

Minh Tran Huy, Les écrivains et le fait divers : une autre histoire de la littérature, Flammarion, 2017.

Jean Milly, 'Un pastiche de Proust: “L'Affaire Lemoine dans les Mémoires de Saint-Simon"', Cahiers Saint-Simon №5, 1977, pp.17-22.

Marcel Proust, 'À propos du "style" de Flaubert' in Chroniques, Gallimard, 1927, pp.193-211.

Jean-Yves Tadié, Marcel Proust, Gallimard, 1996. 